
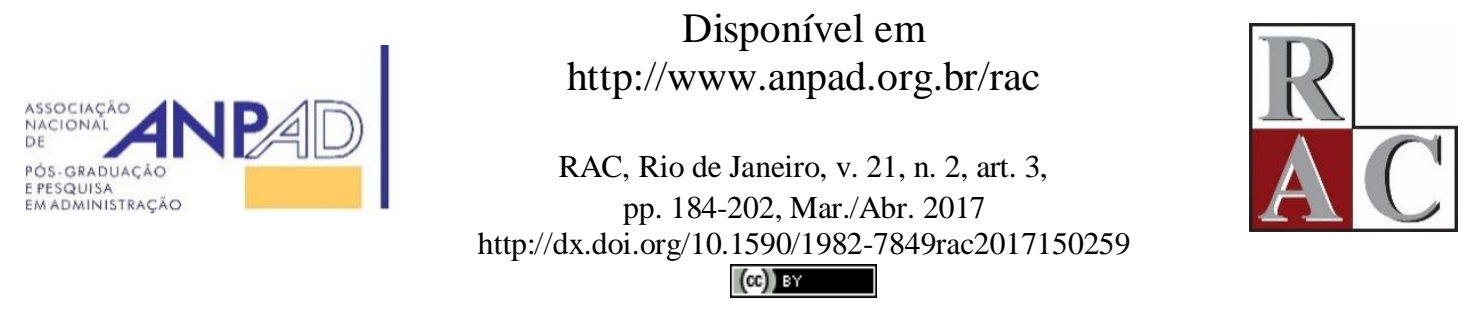

\title{
Tangibilidade e Intangibilidade na Identificação do Desempenho Persistente: Evidências no Mercado Brasileiro
}

Tangibility and Intangibility in Identification of Persistent Performance: Evidence from the Brazilian Stock Market

Ayron Wanderley Medeiros ${ }^{1}$ Anderson Luiz Rezende Mol ${ }^{1}$ Universidade Federal do Rio Grande do Norte ${ }^{1}$

Artigo recebido em 20.09.2015. Última versão recebida em 10.03.2016. Aprovado em 12.03.2016. Publicado online em 06.10.2016. 


\title{
Resumo
}

Carvalho, Kayo e Martin (2010) apontam que o desempenho acima da média e persistente de uma organização pode ser explicado por sua capacidade de explorar adequadamente os recursos e competências tidos como raros e valiosos. Esta pesquisa investigou se a persistência do desempenho superior de companhias abertas brasileiras, por setor, pode ser atribuída à tangibilidade, à intangibilidade, aos níveis de governança corporativa e ao grau de responsabilidade social das companhias. A amostra se constituiu das empresas brasileiras listadas na BM\&FBovespa e as informações foram coletadas na base de dados da consultoria Bloomberg. Utilizou-se uma abordagem de painel dinâmico, por meio do Método dos Momentos Generalizados de Sistema (GMM-SYS) de Arellano e Bover (1995) e Blundell e Bond (1998). Os resultados apontam para a evidência significativa de que a intangibilidade impõe reduções à persistência do desempenho das companhias, na grande maioria dos setores. A tangibilidade e níveis de governança corporativa apresentam efeito heterogêneo sobre o desempenho persistente. Os níveis de responsabilidade social impactam, positivamente e significativamente, a persistência do desempenho superior das companhias abertas no Brasil.

Palavras-chave: ativos; desempenho; persistência.

\begin{abstract}
Carvalho, Kayo and Martin (2010) point out that the above-average performance and persistent of an organization can be explained by its ability to properly exploit the resources and skills seen as rare and valuable. This research investigated if persistence of superior performance by Brazilian companies, by sector, can be attributed to tangibility, intangibility, corporate governance levels and the degree of company social responsibility. The sample consisted of Brazilian companies listed on the BM\&F Bovespa Brazilian stock exchange and information was collected from the Bloomberg consulting database. We used a dynamic panel approach using the System Generalized of Moments Method (GMM-SYS) from Arellano and Bover (1995) and Blundell and Bond (1998). The results indicate significant evidence that intangibility imposed reductions to the persistence of company performance in most sectors. Tangibility and corporate governance levels have heterogeneous effects on persistent superior performance. Social responsibility levels positively and significantly impact the persistence of superior performance of public companies in Brazil.
\end{abstract}

Key words: assets; performance; persistence. 


\section{Introdução}

Nos últimos anos, as empresas têm priorizado recursos que lhes garantam vantagens competitivas ao longo do tempo, no intuito de maximizar seu valor. Esses recursos as auxiliam a alcançar retornos acima da média e permitem criar e sustentar seu valor econômico (Kayo, Kimura, Martin, \& Nakamura, 2006). Organizar a estrutura dos recursos e associá-los ao desempenho financeiro é base para dar suporte à estratégia de competitividade, defendida pela Resource Based View (RBV) (Barney, 1991; Lockett, Morgenstern, \& Thompson, 2009).

Neste ponto, Wernerfelt (1984) aponta que os recursos disponíveis às companhias se distribuem entre ativos tangíveis e intangíveis. O lócus de discussão subjacente à abordagem da Visão Baseada em Recursos (VBR) é que o propulsor das diferenças de desempenho entre as empresas está na capacidade dessas mesmas de se distinguirem dos seus competidores, por meio da alocação e uso estratégico dos ativos tangíveis e intangíveis que proporcionariam, em tese, vantagens competitivas, sob o argumento de que os competidores e concorrentes teriam dificuldade de reproduzir os benefícios de tais recursos.

O reflexo das decisões de investimento de capital, em específico os recursos tangíveis, pode influenciar na obtenção de desempenho superior das empresas e o valor de mercado da ação, como evidencia o estudo de Lucchesi e Famá (2007), para o mercado brasileiro. Nesta mesma linha, os trabalhos de Mcconnel e Muscarella (1985), Chung, Wright e Charoenwong (1999), Burton, Lonie e Power (1999), Lyra e Olinquevitch (2007) verificaram, de forma geral, uma relação desse desempenho com os ativos tangíveis.

Por outro lado, no Brasil, Kayo, Kimura, Martin e Nakamura (2006) e Carvalho, Kayo e Martin (2010) apontam que, à medida que as companhias tiveram acesso facilitado a ativos tangíveis, a posse e uso de ativos intangíveis passou a ser determinante fator de diferenciação, sobretudo em função da dificuldade de sua obtenção e pelas suas especificidades. As investigações contemporâneas defendem a proposição de que esses ativos são os principais drivers de aumento de valor de mercado nas firmas e são endossados pelos trabalhos de Lev (2001) Lev e Zambon (2003), Daun (2003), Carmeli e Tishler (2004), Bianchi e Labory (2004), Villalonga (2004), Cohen (2005), Chareonsuk e Chansa-Ngavej (2008), Colauto, Nascimento, Avelino e Bispo (2009), Lin e Tang (2009) e Surroca, Tribó e Waddock (2010).

Considerando um racional diagnóstico de empresa e a precificação de ativos, os resultados do estudo de Danthine e Jin (2007) indicaram que as propriedades para avaliação e preços das ações são muito dependentes dos ativos intangíveis, pois estes são os resultados dos investimentos em novos produtos, publicidade, inovação e pesquisa, além da capacitação dos funcionários no labor.

Entretanto, Santos, Basso, Kimura e Kayo (2014) avaliaram o esforço inovador, o capital empregado e o capital humano sobre a lucratividade de empresas brasileiras. De forma geral, evidenciaram que os investimentos em inovação não explicam, de modo relevante, o desempenho financeiro das companhias, embora a teoria e estudos internacionais defendam a importância dessas aplicações para o sucesso ou desempenho financeiro superior dessas corporações.

Com o propósito de identificar se os recursos tidos como valiosos, inimitáveis, raros e organizáveis estão associados à vantagem competitiva sustentável, Pavão, Sehnem e Hoffmann (2011) encontraram que os recursos desenvolvidos internamente geram uma consolidada sustentabilidade na vantagem competitiva. Entretanto, para setores da economia brasileira, as evidências em Carvalho et al. (2010) apontam para a predominância de recursos tangíveis.

Assim, as investigações acerca da relevância dos ativos tangíveis e intangíveis, como elemento diferenciador na criação de valor para as companhias, têm se mostrado difusas e dissonantes na literatura internacional. 
Isto posto, este estudo propõe investigar se a estrutura de recursos tangíveis e intangíveis, como recomenda Pavão et al. (2011), é capaz de explicar o desempenho persistente nas companhias de capital aberto no Brasil. Adicionalmente, a investigação incorpora a sugestão de Carvalho et al. (2010), no que se refere à atualização da janela de dados, realizando o uso dos relatórios contábeis no padrão International Financial Reporting Standards (IFRS).

De forma geral, este trabalho assume contornos de atualização dos estudos de Carvalho et al. (2010), no sentido da proposta central de investigação. Além disso, o presente trabalho amplia tal proposta por incorporar no modelo de análise o controle para os efeitos da Responsabilidade Social e da Governança Corporativa sobre o desempenho persistente das firmas. Tal iniciativa procura caracterizar, com maior robustez, os determinantes da persistência no desempenho das companhias de capital aberto no Brasil.

Vale mencionar que evidências sobre a determinância da responsabilidade social sobre o desempenho financeiro das companhias são amplamente discutidas em Wood (1991), Pava e Krausz (1996), Orlitzky (2001), Mackey, Mackey e Barney (2005), Brammer, Brooks e Pavelin (2006) e Laan, Ees e Witteloostuijn (2008).

No Brasil, Ferreira, Ávila e Faria (2010) apontam que, recentemente, a responsabilidade social tem ganhado importância e relevância teórico-empírica. Observa-se uma transformação no próprio conceito, antes, baseado na caridade e altruísmo e, atualmente, definido como uma função de organização transformadora da sociedade. Em contrapartida, os achados de Freguette, Nossa e Funchal (2014) e M. R. Machado, Machado e Corrar (2009) têm apontado para a não evidência de que os retornos do índice de carteira composta de corporações com sustentabilidade social (ISE-BMFBovepa) difiram dos retornos médios de outras carteiras (Ibovespa, IBRX, etc.).

Portanto, existe certa dissonância e controvérsia acerca dos efeitos da responsabilidade social no desempenho das companhias abertas no Brasil. Adicionalmente, esta investigação assume formas ainda mais relevantes, por conduzir esforços de maneira a compreender a influência da responsabilidade social sobre o fenômeno do desempenho persistente.

Em relação aos efeitos da governança corporativa sobre o desempenho, Leal (2004) explica que pesquisas recentes sugerem que a governança corporativa está alicerçada no maior valor e no desempenho dessas firmas, em linha com os estudos de Shleifer e Vishny (1997), Kapler e Love (2002) e Leal e Carvalhal da Silva (2007). Os vários achados acerca do tema apontam, conforme Catapan e Colauto (2014), para uma relação significativa positiva entre o desempenho e os níveis de governança corporativa.

Diante do exposto, pretende-se contribuir para o estado da arte sobre o tema, à medida que se revisam e ampliam os achados de Carvalho et al. (2010), adicionando novos determinantes para o desempenho financeiro persistente das companhias brasileiras à luz do nível de governança corporativa e do grau de responsabilidade social.

\section{Referencial Teórico}

Uma entidade empresarial é um conjunto de recursos que fundamentam o seu desempenho diferenciado de outras entidades. Dessa forma, ela é formalizada como o resultado das potencialidades e restrições de sua carteira de recursos, e não apenas da carteira de produtos, até porque o produto é o resultado da combinação dos recursos (Barney, 1991).

Isso direciona o mainstream da RBV ou Visão Baseada em Recursos, a qual, no campo estratégico, mostra que as empresas modelam uma combinação adequada dos seus recursos de forma heterogênea, em cada setor de atuação, devido ao dinamismo dos processos e aos limites impostos à capacidade produtiva (Mathews, 2002). 
Todavia, existe um modelo denominado Value, Rarity, Imitability and Organisation (VRIO), que defende que os recursos e as competências, sozinhos, não asseguram o desempenho superior, pois este é atingido quando atendem a determinadas condições, como ser valorosos, raros e difíceis, sendo que a empresa deve possuir condições organizacionais para explorar as rendas geradas por tais recursos (Barney \& Hesterley, 2007).

Os recursos são todos os ativos que encadeiam processos organizacionais, capacidades, atributos das firmas, informações, entre outros, controlados pela empresa em que constituem elementos básicos para o desenvolvimento dos serviços e produtos (Kunc \& Morecroft, 2010). Conforme Brito e Vasconcelos (2004), as empresas são vistas como um conjunto de ativos tangíveis e intangíveis, cuja combinação específica resulta nas capacidades competitivas. Com a natureza tácita das capacidades organizacionais e as sinergias, explicam o potencial gerador de diferenças sustentáveis de desempenho.

O desempenho superior da firma, em relação aos seus rivais, está relacionado com a vantagem competitiva. Essa obtenção se dá por meio da utilização de recursos ou capacidades específicas que permitem redundar retornos financeiros superiores, caracterizados como um desempenho persistente ou sustentável em longo prazo (Carvalho, Kayo, \& Martin, 2010).

O estudo de Pavão et al. (2011) corrobora a inferência de que os recursos organizacionais permitem obter uma vantagem competitiva sustentável perante os concorrentes por meio da melhor combinação possível, pois eles interagem entre si, influenciando e sendo influenciados pelo conjunto controlado da empresa.

Nesse raciocínio, a RBV tece o conceito de vantagem competitiva sustentável como o desempenho persistente, e estabelece quando uma empresa vem a adquiri-la, como um encadeamento dos recursos em conjunto que sustentam essa posição como fonte diferencial de competitividade. Essa sustentação é possível em função da forma como se usam os recursos estratégicos e em função da obtenção dos lucros superiores (Barney, 1991).

Os autores Carvalho et al. (2010) consideram os ativos como um núcleo central para explicar o desempenho superior. Eles são vistos como tangíveis e intangíveis e entendidos como uma unidade fundamental de geração de valor.

Pavão et al. (2011) enfatizam a estrutura de ativos que devem permitir uma dinâmica para que se atinja a eficiência e a eficácia. Então, a tangibilidade e intangibilidade, caracterizadas como estrutura, são vistas em conjunto, como fatores que influenciam diretamente os resultados das organizações.

A diversidade de abordagens, na discussão da vantagem competitiva, se relaciona diretamente com a atuação superior da empresa em relação às suas rivais. $\mathrm{O}$ desempenho obtido, por meio da utilização de recursos ou capacidades específicas indisponíveis aos concorrentes, permite retornos financeiros superiores em relação a estes concorrentes.

Neste estudo, é adotada a proposta inserida por Peteraf (1993) e também utilizada por Carvalho et al. (2010), que considera a obtenção de vantagem competitiva o resultado financeiro superior ao da média do setor de atividades do qual a empresa participa.

Sabe-se que resultados conflitantes, por razão das medidas do desempenho, são apresentados em vários estudos, a exemplo de Glick Washburn e Miller (2005). De acordo com esses autores, uma das possíveis causas é a pobreza na conceituação, operacionalização e mensuração do constructo para medir o desempenho, o que traz inconsistências nos resultados.

Entretanto, a consecução da vantagem competitiva não é condição suficiente para garantir uma performance superior da empresa. É necessário que haja sustentabilidade e persistência como condição essencial. Então, como adotado em Carvalho et al. (2010), o desempenho persistente é observado em uma janela de tempo. 
A diversidade dos recursos e competências é a base para a vantagem competitiva sustentável. Mathews (2002) enfatiza que os recursos controlados pelas empresas explicam esse desempenho e são entendidos como sendo a unidade fundamental de geração de valor pelas companhias.

\section{Procedimentos Metodológicos}

Como referência para o processo de construção das variáveis, utilizou-se o artigo de Carvalho et al. (2010). Consequentemente, a pesquisa seguiu em explicar se o desempenho persistente guarda relação com a intangibilidade, a partir do Q de Tobin (medida aplicada por Villalonga, 2004), ou com a tangibilidade dos recursos, através do uso da variável que mede a variação do patrimônio líquido (medida utilizada em Carvalho et al., 2010; Daniel \& Titman, 2006).

Esta pesquisa investigou as empresas brasileiras de capital aberto, com ações negociadas na Bolsa de Valores, Mercadorias e Futuros (BM\&FBovespa). Extraíram-se os dados por meio da plataforma Bloomberg.

Empregou-se a divisão setorial utilizada pela BM\&FBovespa. O número de companhias em cada setor é apresentado na Tabela 1. Foram excluídas as empresas do setor Financeiro, caracterizadas como bancos e instituições financeiras, em decorrência da peculiaridade de suas operações e de relatórios contábeis, e também se eliminou aquelas com patrimônio líquido negativo, por se presumir problemas de solvência financeira. Estas exclusões têm se mostrado consistentes e foram adotadas por Fama e French (1993), Villalonga (2004) e Daniel e Titman (2006).

Tabela 1

\section{Classificação Setorial}

\begin{tabular}{ll}
\hline Setor & Quantidade de empresas \\
\hline Materiais básicos & 13 \\
Bens industriais & 6 \\
Construção e transporte & 13 \\
Consumo não cíclico & 12 \\
Consumo cíclico & 9 \\
Telecomunicações e tecnologia & 6 \\
Utilidade pública & 26 \\
\hline Total & $\mathbf{8 5}$ \\
\hline
\end{tabular}

Nota. Fonte: Dados da pesquisa.

O período de análise adotado compreendeu os anos de 2008 a 2014, por representar o padrão IFRS já adotado pelas empresas brasileiras de capital aberto. As empresas que não oportunizaram todas as informações necessárias para o estudo e em todos os anos do período da análise foram eliminadas.

\section{Descrição das variáveis}

De forma sucinta, as variáveis utilizadas estão evidenciadas na Tabela 2: 
Tabela 2

\section{Descrição Sucinta das Variáveis}

\begin{tabular}{lcl}
\hline Tipo & Variável & Descrição \\
\hline Regressando & LEF & Medida de desempenho \\
\hline Regressor & $L E F_{t-1}$ & Medida de desempenho defasado em um lag \\
\hline Regressor & QTobin & Medida de intangibilidade \\
\hline Regressor & VarBook & Medida de tangibilidade \\
\hline Regressor & ESG & Indicador Bloomberg do grau de responsabilidade social \\
\hline Regressor & Govnce & Indicador Bloomberg do nível de governança \\
\hline Regressor & QTobin $\times L E F_{t-1}$ & Variável de Interação da medida de intangibilidade e desempenho defasado \\
\hline Regressor & VarBook $x L E F_{t-1}$ & Variável de Interação da medida de tangibilidade e desempenho defasado \\
\hline Regressor & $E S G \times L E F_{t-1}$ & Variável de Interação do indicador de responsabilidade social e desempenho \\
& defasado \\
\hline Regressor & Govnce $\times L E F_{t-1}$ & Variável de Interação do indicador de governança e desempenho defasado \\
\hline Regressor & $L E F_{t-1}{ }^{2}$ & Termo quadrático da medida de desempenho defasado em um lag \\
\hline
\end{tabular}

Nota. Fonte: Dados da pesquisa.

LEF: lucro específico da firma é a medida do desempenho da empresa, conforme modelo de Villalonga (2004), calculado pela diferença entre o ROA da firma e o ROA médio do setor da economia, do qual a empresa faz parte no ano $t$. A formalização do procedimento está explícita na equação 1:

$$
L E F_{i, t}=R O A_{i, t}-\left(\sum_{\mathrm{i}=1}^{i, j, t} \frac{R O A_{i, j, t}}{I_{j, t}}\right)
$$

Onde: $I_{j, t}=$ número de empresas com dados disponíveis do setor $j$ no ano $t ; i$ e $t$ representam empresa e tempo.

QTobin: medida que representa o grau de intangibilidade da firma, conforme modelo de Villalonga (2004), sendo calculado conforme equação 2:

$$
\text { QTobin }=\frac{V M+D T}{A T}
$$
total.

Onde: $\mathrm{VM}=$ valor de mercado da empresa; $\mathrm{DT}$ = dívidas totais contábeis da empresa; $\mathrm{AT}$ = ativo

ESG: variável de controle para o grau de responsabilidade social da empresa. Mensurada pela consultoria Bloomberg.

Govnce: variável de controle para o nível de governança corporativa da companhia. Mensurada pela consultoria Bloomberg.

As variáveis explicativas foram transformadas para seu Logaritmo Natural de forma a atenuar uma possível concentração em distribuições condicionais advindas de variáveis estritamente positivas, além de suavizar uma possível heterocedasticidade. 


\section{Modelo econométrico}

Neste estudo, os coeficientes serão aferidos a partir dos dados de painel balanceados. Apresentamse resultados econométricos para os estimadores de dados em painel, por meio do GMM de sistema de Arellano e Bover (1995) e Blundell e Bond (1998) para abordagens dinâmicas. A utilização da abordagem dinâmica se justifica devido às características de memória subjacente à persistência. Tal estrutura permite capturar o mecanismo de ajustamento da variável dependente conforme apontado em Greene (2003) e Baltagi (2008). Adicionalmente, o modelo dinâmico reproduz estimadores consistentes na presença de endogeneidade pelo fato de não tratar as variáveis do modelo como estritamente exógenas. Baltagi (2008) aponta a omissão de variáveis, a seletividade da amostra e erros de medida como os principais geradores de endogeneidade.

As proposições de Arellano e Bond (1991) e de Blundell e Bond (1998) empregam procedimentos com variáveis instrumentais que contornam a possível endogeneidade do modelo. Estes procedimentos requerem o teste de Sargan de restrição sobreidentificada, de forma a verificar a validade dos instrumentos utilizados. No caso de modelos utilizando amostras finitas, a estimação consistente é obtida através do estimador GMM-SYS de dois estágios com erros padrão robustos, corrigidos da heterocedasticidade. Neste caso, as estimativas de Sargan são desnecessárias (Windmeijer, 2005).

Nas formulações dinâmicas com dados em painel, como no presente estudo, a correlação existente entre a variável dependente defasada $y_{i, t-1}$ e o termo de erro $\varepsilon_{i, t}$ imputa endogeneidade ao modelo, sendo necessário especificar uma transformação em primeira diferença às variáveis, o que cria dificuldades no tratamento de modelos de efeitos fixos e aleatórios, pois remove o termo constante e o termo de efeitos individuais.

A natureza dinâmica no processo de estimação será captada pela utilização da variável dependente com defasagem em um período como variável explicativa. A definição da ordem de defasagem se deu pelo teste de correlação serial nos resíduos dos modelos estimados, confirmado pelo Critério de Informação de Akaike (comparado com modelos com defasagens mais elevadas).

Em razão de as variáveis defasadas serem muitas vezes instrumentos pobres para as primeiras diferenças das variáveis (utilizadas nos modelos dinâmicos), especialmente se estas estão próximas de um passeio aleatório, o procedimento usado para estimar o painel dinâmico é baseado em Arellano e Bover (1995) e Blundell e Bond (1998). O estimador é comumentemente conhecido como GMM de sistema (GMM-SYS).

No GMM-SYS, estima-se a equação de primeiras diferenças e incorporam-se as defasagens das variáveis em nível como instrumentos para a variável endógena. Esse procedimento reproduz instrumentos mais consistentes no processo de modelagem, obtendo-se um conjunto adicional de condições de momento que contribui com a melhoria das propriedades de pequena amostra do estimador, principal crítica do estimador clássico de Arellano e Bond (1991).

Por construção, o modelo GMM-SYS é sobreidentificado para painéis com período de tempo maior que 3, visto que se usam todas as defasagens disponíveis como potenciais condições de momento. À medida que avançamos para os períodos posteriores em séries de tempo de cada painel, condições de ortogonalidade adicionais se tornam disponíveis e, tendo essas condições adicionais em conta, melhora a eficiência do estimador. Neste sentido, as variáveis instrumentais compõem-se de todas as variáveis explicativas em diferenças defasadas de até três lags. Nesta pesquisa, os instrumentos foram defasados de 2 a 3 períodos. As evidências econométricas apontadas na literatura convergem para defasagens entre dois e cinco lags. A utilização de lags mais amplos potencializam efeitos indesejados de over-fitting.

Os modelos econométricos utilizados para a intangibilidade, eq. (3), e tangibilidade, eq. (4), são representados a seguir: 


$$
\begin{aligned}
& L E F_{i, t}=\alpha_{i}+\beta_{1} L E F_{i, t-1}+\beta_{2} \text { QTobin }_{i, t}+\beta_{3} E S G_{i, t}+\beta_{4} \text { Govnce }_{i, t}+ \\
& \beta_{5}\left(L E F_{i, t-1} \text { QTobin }_{i, t}\right)+\beta_{6}\left(L E F_{i, t-1} E S G_{i, t}\right)+\beta_{7}\left(L E F_{i, t-1} \text { Govnce }_{i, t}\right)+\beta_{8}\left(L E F_{i, t-1}\right)^{2}+ \\
& \mu_{\mathrm{i}}+\varepsilon_{\mathrm{i}, \mathrm{t}}
\end{aligned}
$$

Onde: $L E F_{i, t-1}=$ lucro específico da empresa defasado; $\beta_{1}=$ captura o efeito do lucro específico da empresa defasado; QTobin $_{i, t}=$ Proxy para intangibilidade; $\beta_{2}=$ captura o efeito do QTobin; $E S G_{i, t}=E S G$ Disclosure $; \beta_{3}=$ captura o efeito do ESG; Govnce ${ }_{i, t}=$ Governance Disclosure $; \beta_{4}=$ captura o efeito do Governance Disclosure; $\beta_{5}=$ captura o efeito de interação entre o lucro específico da empresa defasado e QTobin (intangibilidade); $\beta_{6}=$ captura o efeito de interação entre o lucro específico da empresa defasado e o ESG Disclosure; $\beta_{7}=$ captura o efeito de interação entre o lucro específico da empresa defasado e o Governance Disclosure; $\beta_{8}=$ captura o possível efeito não linear do lucro específico da empresa defasado em (t-1) sobre o lucro específico da empresa em $\mathrm{t} ; \mu_{i}=$ efeito específico da firma que não varia com o tempo; $\varepsilon_{i, t}=$ termo de erro da firma.

$$
\begin{aligned}
& L E F_{i, t}=\alpha_{i}+\beta_{1} L E F_{i, t-1}+\beta_{2} \operatorname{Varbook}_{i, t}+\beta_{3} E S G_{i, t}+\beta_{4} \text { Govnce }_{i, t}+ \\
& \beta_{5}\left(L E F_{i, t-1} \operatorname{Varbook}_{i, t}\right)+\beta_{6}\left(L E F_{i, t-1} E S G_{i, t}\right)+\beta_{7}\left(L E F_{i, t-1} \text { Govnce }_{i, t}\right)+\beta_{8}\left(L E F_{i, t-1}\right)^{2}+ \\
& \mu_{\mathrm{i}}+\varepsilon_{\mathrm{i}, \mathrm{t}}
\end{aligned}
$$

Onde: $L E F_{i, t-1}=$ lucro específico da empresa defasado; $\beta_{1}=$ captura o efeito do lucro específico da empresa defasado; $\operatorname{Varbook}_{i, t}=$ Proxy para tangibilidade; $\beta_{2}=$ captura o efeito do QTobin; $E S G_{i, t}=E S G$ Disclosure $; \beta_{3}=$ captura o efeito do ESG; Govnce $_{i, t}=$ Governance Disclosure $; \beta_{4}=$ captura o efeito do Governance Disclosure; $\beta_{5}=$ captura o efeito de interação entre o lucro específico da empresa defasado e Varbook (tangibilidade); $\beta_{6}=$ captura o efeito de interação entre o lucro específico da empresa defasado e o ESG Disclosure; $\beta_{7}=$ captura o efeito de interação entre o lucro específico da empresa defasado e o Governance Disclosure; $\beta_{8}=$ captura o efeito não linear do lucro específico da empresa defasado; $\mu_{i}=$ efeito específico da firma que não varia com o tempo; $\varepsilon_{i, t}=$ termo de erro da firma.

\section{Análise dos Resultados}

\section{Relação entre desempenho persistente e intangibilidade, governança e responsabilidade social}

A Tabela 3 sumariza a relação do lucro específico da firma (LEF) contra o lucro específico defasado $\left(L E F_{t-1}\right)$, a variável da intangibilidade (QTobin) e a variável de responsabilidade social (ESG) e de governança corporativa (Govnce). 
Tabela 3

Intangibilidade, Governança e Responsabilidade Social e Desempenho Persistente das Companhias Brasileiras

\begin{tabular}{|c|c|c|c|c|c|c|c|}
\hline & $\begin{array}{l}\text { Bens } \\
\text { Industriais }\end{array}$ & $\begin{array}{l}\text { Construção } \\
\text { e Transporte }\end{array}$ & $\begin{array}{l}\text { Consumo } \\
\text { Cíclico }\end{array}$ & $\begin{array}{l}\text { Consumo } \\
\text { Não Cíclico }\end{array}$ & $\begin{array}{l}\text { Materiais } \\
\text { Básicos }\end{array}$ & $\begin{array}{l}\text { Telecom. e } \\
\text { Tecnologia }\end{array}$ & $\begin{array}{l}\text { Utilidade } \\
\text { Pública }\end{array}$ \\
\hline Constante & $\begin{array}{l}-94,6362 * * * \\
{[32,0893]}\end{array}$ & $\begin{array}{l}-72,0824 * * * \\
{[19,3158]}\end{array}$ & $\begin{array}{l}-63,5674 * * * \\
{[21,5534]}\end{array}$ & $\begin{array}{l}-48,4389 * * * \\
{[19,0345]}\end{array}$ & $\begin{array}{l}-15,6944 * * \\
{[6,7863]}\end{array}$ & $\begin{array}{l}33,1359 \\
{[22,8463]}\end{array}$ & $\begin{array}{l}5,0438 * * * \\
{[2,1255]}\end{array}$ \\
\hline $\mathrm{LEF}_{\mathrm{t}-1}$ & $\begin{array}{l}2,0413 * * \\
{[0,8972]}\end{array}$ & $\begin{array}{l}7,5555^{* *} \\
{[3,2309]}\end{array}$ & $\begin{array}{l}5,5669 * * \\
{[2,5269]}\end{array}$ & $\begin{array}{l}1,04430 * * \\
{[0,4734]}\end{array}$ & $\begin{array}{l}-0,07725 \\
{[0,0594]}\end{array}$ & $\begin{array}{l}-0,5890 \\
{[1,0594]}\end{array}$ & $\begin{array}{l}4,0004 * * \\
{[2,0019]}\end{array}$ \\
\hline QTobin & $\begin{array}{l}-1,939793 * * * \\
{[1,5742]}\end{array}$ & $\begin{array}{l}7,3153 * * \\
{[3,3835]}\end{array}$ & $\begin{array}{l}2,3066 * * \\
{[1,0426]}\end{array}$ & $\begin{array}{l}3,4262 * * \\
{[1,5834]}\end{array}$ & $\begin{array}{l}8,7376 * * * \\
{[3,1385]}\end{array}$ & $\begin{array}{l}55,869 * * \\
{[21,8631]}\end{array}$ & $\begin{array}{l}4,4184 * * * \\
{[1,3039]}\end{array}$ \\
\hline ESG & $\begin{array}{l}8,3436 * * * \\
{[2,2636]}\end{array}$ & $\begin{array}{l}10,1867 * * * \\
{[2,5950]}\end{array}$ & $\begin{array}{l}23,6283 * * \\
{[10,4120]}\end{array}$ & $\begin{array}{l}13,9052 * * * \\
{[4,4135]}\end{array}$ & $\begin{array}{l}3,1114 * * \\
{[1,5928]}\end{array}$ & $\begin{array}{l}69,7633 * * * \\
{[19,4625]}\end{array}$ & $\begin{array}{l}3,6138 * * * \\
{[1,8953]}\end{array}$ \\
\hline Govnce & $\begin{array}{l}19,6116 \\
{[9,1186]}\end{array}$ & $\begin{array}{l}8,5467 \\
{[10,2095]}\end{array}$ & $\begin{array}{l}7,2265 \\
{[14,3734]}\end{array}$ & $\begin{array}{l}-3,4627 * * * \\
{[1,3139]}\end{array}$ & $\begin{array}{l}-1,9795 \\
{[3,2847]}\end{array}$ & $\begin{array}{l}-102,9075^{* *} \\
{[50,3482]}\end{array}$ & $\begin{array}{l}-6,9190 * * \\
{[3,0793]}\end{array}$ \\
\hline QTobin $x$ LEF $_{t-1}$ & $\begin{array}{l}-0,4758^{* * * *} \\
{[0.0826]}\end{array}$ & $\begin{array}{l}-0,3761 * * * \\
{[0,1393]}\end{array}$ & $\begin{array}{l}-0,9952 * * * \\
{[0,0858]}\end{array}$ & $\begin{array}{l}-0,1172 * * * \\
{[0,0202]}\end{array}$ & $\begin{array}{l}-0,5873 * * * \\
{[0,1614]}\end{array}$ & $\begin{array}{l}-0,4440 * * \\
{[0,13153]}\end{array}$ & $\begin{array}{l}0,6710 * * * \\
{[0,2185]}\end{array}$ \\
\hline ESG $x$ LEF $_{t-1}$ & $\begin{array}{l}0,0010^{* * * *} \\
{[0,0007]}\end{array}$ & $\begin{array}{l}2,2087 * * \\
{[0,9876]}\end{array}$ & $\begin{array}{l}1,8212 * * \\
{[0,8340]}\end{array}$ & $\begin{array}{l}0,5693 * * \\
{[0,2473]}\end{array}$ & $\begin{array}{l}0,4965 * * * \\
{[0,1268]}\end{array}$ & $\begin{array}{l}0,3945 * * \\
{[0,1621]}\end{array}$ & $\begin{array}{l}0,4943 * * \\
{[0,2070]}\end{array}$ \\
\hline Govnce $x L^{2 E F} F_{t-1}$ & $\begin{array}{l}-0,05320 * * \\
{[0,2484]}\end{array}$ & $\begin{array}{l}-4,6818 \\
{[4,2009]}\end{array}$ & $\begin{array}{l}0,01646 \\
{[0,0356]}\end{array}$ & $\begin{array}{l}0,6697 * * \\
{[0,3236]}\end{array}$ & $\begin{array}{l}-0,3364 \\
{[0,2856]}\end{array}$ & $\begin{array}{l}0,0943 \\
{[0,1543]}\end{array}$ & $\begin{array}{l}-1.8223 * * * \\
{[0,6485]}\end{array}$ \\
\hline $\mathrm{LEF}_{\mathrm{t}-1}{ }^{2}$ & $\begin{array}{l}0,01243 \\
{[0,1113]}\end{array}$ & $\begin{array}{l}0,0241 * * \\
{[0,0100]}\end{array}$ & $\begin{array}{l}0,0220 * * * \\
{[0,0080]}\end{array}$ & $\begin{array}{l}0,0180 * * * \\
{[0,0058]}\end{array}$ & $\begin{array}{l}0,0012 \\
{[0,0096]}\end{array}$ & $\begin{array}{l}0,0012 \\
{[0,0018]}\end{array}$ & $\begin{array}{l}0,0130 * * \\
{[0,0053]}\end{array}$ \\
\hline Prob $>\mathrm{Chi}^{2}$ & 0,0000 & 0,0000 & 0,0000 & 0,0000 & 0,0000 & 0,0000 & 0,0000 \\
\hline Sargan & $\begin{array}{l}\mathrm{Chi}^{2}(8)= \\
11.5032 \\
(0,1748)\end{array}$ & $\begin{array}{l}\mathrm{Chi}^{2}(8)= \\
6,6991 \\
(0,5694)\end{array}$ & $\begin{array}{l}\mathrm{Chi}^{2}(8)= \\
5,1563 \\
(0,7407)\end{array}$ & $\begin{array}{l}\mathrm{Chi}^{2}(8)= \\
3,2250 \\
(0,9194)\end{array}$ & $\begin{array}{l}\mathrm{Chi}^{2}(8)= \\
6,3303 \\
(0,6103)\end{array}$ & $\begin{array}{l}\mathrm{Chi}^{2}(8)= \\
2,37 \mathrm{e}-22 \\
(1,0000)\end{array}$ & $\begin{array}{l}\mathrm{Chi}^{2}(8)= \\
7,3399 \\
(0,4994)\end{array}$ \\
\hline AIC & 4,1134 & 4,0876 & 3,3331 & 3,6502 & 4,1101 & 3,8993 & 4,0855 \\
\hline $\begin{array}{l}\text { Defasagens } \\
\text { Instrumentos }\end{array}$ & 2 a 3 & 2 a 3 & 2 a 3 & 2 a 3 & 2 a 3 & 2 a 3 & 2 a 3 \\
\hline AR(1) (Prob.) & 0,0128 & 0,0028 & 0,0097 & 0,0044 & 0,0073 & 0,0126 & 0,0013 \\
\hline AR(2) (Prob.) & 0,8308 & 0,6985 & 0,7833 & 0,8819 & 0,5819 & 0,7661 & 0,9579 \\
\hline
\end{tabular}

Nota. Fonte: elaborada pelos autores. Estimativas obtidas através do método GMM-SYS em dois estágios; Erros padrão robustos entre colchetes; teste Sargan implementado com erros GMM. Probabilidade do teste Sargan entre parênteses. AR(1) e $\mathrm{AR}(2)$ representam probabilidades dos testes de correlação serial de primeira e segunda ordem.

$*, * *$ e $* * *$ denotam, respectivamente, significância de $10 \%, 5 \%$ e $1 \%$.

Observa-se que o coeficiente que mede a persistência do desempenho superior $\left(L E F_{t-1}\right)$, para os diversos setores, apresentou significância estatística a 5\% e com sinal positivo, com a indicação de que o lucro específico da firma em t-1 contribui para formação do lucro específico da firma em t. Tal característica corrobora as evidências em Carvalho et al. (2010). Neste estudo citado, a amostra, composta por companhias com vantagem competitiva, confirma o efeito significativo a $5 \%$, com sinal positivo para a persistência do LEF.

Em relação ao efeito da intangibilidade sobre o desempenho das companhias, observa-se um efeito positivo e significativo de pelo menos $5 \%$ para todos os ramos, com exceção do setor de Bens Industriais, que apresentou sinal negativo para seu coeficiente. $O$ efeito da interação do lucro específico, defasado com a intangibilidade, exibiu-se significativo para todos os setores e comprova os indícios 
encontrados em Carvalho et al. (2010). Assim, estes sugerem que a intangibilidade dos recursos concorre, significativamente, para reduzir a persistência do desempenho superior, em todos os setores investigados, exceto o de Utilidade Pública, em que a evidência converge para confirmar o desempenho superior.

Os resultados deste artigo divergem de Villalonga (2004) para empresas norte-americanas. Os resultados deste artigo citado investigação demonstraram que a intangibilidade dos ativos tem um papel relevante para a persistência do desempenho superior. Sua conclusão é construída sob o argumento de que a variável de interação (QTobin $\mathrm{x} L E F_{t-1}$ ) apresenta relação positiva e significativa com o lucro contemporâneo $\left(L E F_{t}\right)$. Isto posto, quanto maior a intangibilidade e lucro específico de uma firma no ano anterior, maior o lucro no ano corrente. Ainda, para os setores da Construção e Transporte, Consumo Cíclico, Consumo Não Cíclico e Utilidade Pública, o $L E F_{t-1}$ apresenta um efeito quadrático positivo sobre o lucro específico contemporâneo.

O indicador de responsabilidade social (ESG) mostrou-se significativo a 1\% de probabilidade, e com relação positiva, para todos os setores analisados, com o lucro específico da firma. Ainda, exibe,

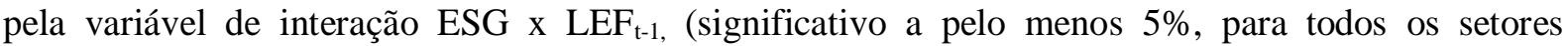
analisados), que maiores indicadores de responsabilidade social e sustentabilidade, associados com maior lucro específico, no ano anterior, contribuem para elevar a persistência do lucro específico dessas entidades no mercado brasileiro. Tal evidência adiciona novas contribuições empíricas, uma vez que se alinha aos achados de M. A. V. Machado e Machado (2011), ao evidenciar que a responsabilidade social contribui para o desempenho das companhias abertas no Brasil. Neste sentido, os achados apontam para o efeito da responsabilidade social, não somente sobre o lucro específico contemporâneo da firma, mas, de modo equivalente, para a sua persistência.

O indicador de governança (Govnce) apresentou-se significativo apenas para os setores de Consumo Não Cíclico, Telecomunicação e Tecnologia e Utilidade Pública. Os dados ratificam para uma relação inversa do índice de governança com o lucro específico das companhias para os setores Consumo Não Cíclico e Utilidade Pública. Tais perspectivas encontram explicação a partir da teoria dos custos de agência e assimetria informacional, haja visto que o esforço para reduzir a assimetria informacional incorre em custos que reduzem o lucro dessas corporações. Ainda, a variável de interação Govnce $\mathrm{x} \mathrm{LEF}_{\mathrm{t}-1}$ tem efeito diverso e heterogêneo em setores distintos.

\section{Relação entre desempenho persistente e tangibilidade, governança e responsabilidade social}

Os resultados que tratam da relação entre desempenho persistente e tangibilidade dos recursos das firmas são apresentados na Tabela 4.

Tabela 4

Tangibilidade, Governança, Responsabilidade Social e Desempenho Persistente das Companhias

\begin{tabular}{llllllll}
\hline & $\begin{array}{l}\text { Bens } \\
\text { Industriais }\end{array}$ & $\begin{array}{l}\text { Construção e } \\
\text { Transporte }\end{array}$ & $\begin{array}{l}\text { Consumo } \\
\text { Cíclico }\end{array}$ & $\begin{array}{l}\text { Consumo } \\
\text { Não Cíclico }\end{array}$ & $\begin{array}{l}\text { Materiais } \\
\text { Básicos }\end{array}$ & $\begin{array}{l}\text { Telecom. e } \\
\text { Tecnologia }\end{array}$ & $\begin{array}{l}\text { Utilidade } \\
\text { Pública }\end{array}$ \\
\hline Constante & $-75,1363 * * * *$ & $-45,2496 * *$ & 9,3461 & $-7,4497 * * *$ & $-7,1218^{* * *}$ & $-13,0453$ & $-11,6203 * * *$ \\
& {$[37,7742]$} & {$[20,2631]$} & {$[10,4795]$} & {$[2,2135]$} & {$[2,1679]$} & {$[23,1942]$} & {$[2,7954]$} \\
\hline $\mathrm{LEF}_{\mathrm{t}-1}$ & $10,3339 * *$ & $16,4384 * *$ & $5,4782^{* *}$ & $2,6531 * *$ & $0,1590 * *$ & $0,1468 * * *$ & $1,0816 * * *$ \\
& {$[5,1572]$} & {$[7,3186]$} & {$[2,1849]$} & {$[1,1700]$} & {$[0,0358\}$} & {$[0,0129]$} & {$[0,2999]$} \\
\hline VarBook & $7,7417 * * *$ & $4,8812 * *$ & 3,5603 & $2,5296 * *$ & $1,7355^{* *}$ & $-0,1832$ & $9,7939 * * *$ \\
& {$[2,4966]$} & {$[2,3186]$} & {$[5,4295]$} & {$[1,0226]$} & {$[0,7430]$} & {$[2,1351]$} & {$[0,6791]$} \\
\hline
\end{tabular}


Tabela 4 (continuação)

\begin{tabular}{|c|c|c|c|c|c|c|c|}
\hline & $\begin{array}{l}\text { Bens } \\
\text { Industriais }\end{array}$ & $\begin{array}{l}\text { Construção e } \\
\text { Transporte }\end{array}$ & $\begin{array}{l}\text { Consumo } \\
\text { Cíclico }\end{array}$ & $\begin{array}{l}\text { Consumo } \\
\text { Não Cíclico }\end{array}$ & $\begin{array}{l}\text { Materiais } \\
\text { Básicos }\end{array}$ & $\begin{array}{l}\text { Telecom. e } \\
\text { Tecnologia }\end{array}$ & $\begin{array}{l}\text { Utilidade } \\
\text { Pública }\end{array}$ \\
\hline ESG & $\begin{array}{l}20,2083 * * * \\
{[6,0441]}\end{array}$ & $\begin{array}{l}4,5093 * * * \\
{[1,4868]}\end{array}$ & $\begin{array}{l}2,6620 * * \\
{[1,6013]}\end{array}$ & $\begin{array}{l}6,0430 * * * \\
{[2,05550]}\end{array}$ & $\begin{array}{l}5,0679 * * * \\
{[0,9274]}\end{array}$ & $\begin{array}{l}0,1317 \\
{[4,6987]}\end{array}$ & $\begin{array}{l}0,1614 * * * \\
{[0,0062]}\end{array}$ \\
\hline Govnce & $\begin{array}{l}3,0083^{* * *} \\
{[1,4118]}\end{array}$ & $\begin{array}{l}15,2292 * * * \\
{[2,4321]}\end{array}$ & $\begin{array}{l}2,7772 \\
{[2,1643]}\end{array}$ & $\begin{array}{l}-3,5173 * * * \\
{[0,9399]}\end{array}$ & $\begin{array}{l}-3,2674 * \\
{[1,9764]}\end{array}$ & $\begin{array}{l}-3,7065 \\
{[3,7795]}\end{array}$ & $\begin{array}{l}-0,6086 \\
{[1,8251]}\end{array}$ \\
\hline VarBook x LEF $\mathrm{LE}_{\mathrm{t}-1}$ & $\begin{array}{l}-0,1702 * * * \\
{[0,0175]}\end{array}$ & $\begin{array}{l}-0,8597 * * \\
{[0,3640]}\end{array}$ & $\begin{array}{l}1,8285 * * * \\
{[0,6499]}\end{array}$ & $\begin{array}{l}-0,15313 * * * \\
{[0,0222]}\end{array}$ & $\begin{array}{l}-0,4297 * * * \\
{[0,1626]}\end{array}$ & $\begin{array}{l}0,8976^{* * *} \\
{[0,1233]}\end{array}$ & $\begin{array}{l}-0,4089 * * * \\
{[0,0342]}\end{array}$ \\
\hline ESG $x$ LEF $_{t-1}$ & $\begin{array}{l}0,6063 * * * \\
{[0,0190]}\end{array}$ & $\begin{array}{l}0,6359 * * * \\
{[0,1876]}\end{array}$ & $\begin{array}{l}0,31422 * * * \\
{[0,0275]}\end{array}$ & $\begin{array}{l}0,2286 * * * \\
{[0,0230]}\end{array}$ & $\begin{array}{l}0,3777 * * * \\
{[0,0881]}\end{array}$ & $\begin{array}{l}0,4356^{* * *} \\
{[0,0567]}\end{array}$ & $\begin{array}{l}0,5118 * * \\
{[0,1367]}\end{array}$ \\
\hline Govnce $x L^{2 E F} F_{t-1}$ & $\begin{array}{l}-3,0083^{* *} \\
{[1,4118]}\end{array}$ & $\begin{array}{l}-4,5959 * * \\
{[2,0275]}\end{array}$ & $\begin{array}{l}1,1741 * * * \\
{[0,4302]}\end{array}$ & $\begin{array}{l}0,8859 * \\
{[0,3028]}\end{array}$ & $\begin{array}{l}-0,2865 \\
{[0,3830]}\end{array}$ & $\begin{array}{l}0,9765 \\
{[0,6845]}\end{array}$ & $\begin{array}{l}-0,5780 \\
{[0,3927]}\end{array}$ \\
\hline $\mathrm{LEF}_{\mathrm{t}-1}{ }^{2}$ & $\begin{array}{l}0,0438 * * \\
{[0,0124]}\end{array}$ & $\begin{array}{l}0,0052 \\
{[0,0146]}\end{array}$ & $\begin{array}{l}-0,0015 \\
{[0,0151]}\end{array}$ & $\begin{array}{l}0,1200^{*} \\
{[0,0069]}\end{array}$ & $\begin{array}{l}0,0061 \\
{[0,0199]}\end{array}$ & $\begin{array}{l}0,0022 \\
{[0,0028]}\end{array}$ & $\begin{array}{l}-0,0113^{* * *} \\
{[0,0039]}\end{array}$ \\
\hline Prob $>\mathrm{chi}^{2}$ & 0,0000 & 0,0003 & 0,0000 & 0,0001 & 0,0000 & 0,0034 & 0,0000 \\
\hline Sargan & $\begin{array}{l}\mathrm{Chi}^{2}(8)= \\
2.9932 \\
(0,9347)\end{array}$ & $\begin{array}{l}\mathrm{Chi}^{2}(8)= \\
3,0991 \\
(0,9279)\end{array}$ & $\begin{array}{l}\mathrm{Chi}^{2}(8)= \\
2,3318 \\
(0,9691)\end{array}$ & $\begin{array}{l}\mathrm{Chi}^{2}(8)= \\
2,2465 \\
(0,9725)\end{array}$ & $\begin{array}{l}\mathrm{Chi}^{2}(8)= \\
3,6201 \\
(0,8896)\end{array}$ & $\begin{array}{l}\mathrm{Chi}^{2}(8)= \\
1,4218 \\
(0,9939)\end{array}$ & $\begin{array}{l}\mathrm{Chi}^{2}(8)= \\
3,3394 \\
(0,9112)\end{array}$ \\
\hline AIC & 3,0045 & 4,6754 & 4,8766 & 3,9987 & 5,1123 & 4,5554 & 4,8766 \\
\hline $\begin{array}{l}\text { Defasagens } \\
\text { Instrumentos }\end{array}$ & 2 a 3 & 2 a 3 & 2 a 3 & 2 a 3 & 2 a 3 & 2 a 3 & 2 a 3 \\
\hline AR(1) - (Prob.) & 0,0221 & 0,0172 & 0,0241 & 0,0134 & 0,0122 & 0,0544 & 0,0041 \\
\hline $\mathrm{AR}(2)-$ (Prob.) & 0,9134 & 0,8499 & 0,8721 & 0,8587 & 0,8678 & 0,7870 & 0,8936 \\
\hline
\end{tabular}

Nota. Fonte: elaborada pelos autores. Estimativas obtidas através do método GMM-SYS em dois estágios; Erros padrão robustos entre colchetes; teste Sargan implementado com erros GMM, Probabilidade do teste Sargan entre parênteses. AR(1) e $\mathrm{AR}(2)$ representam probabilidades dos testes de correlação serial de primeira e segunda ordem.

$*, * *$ e $* * *$ denotam, respectivamente, significância de $10 \%, 5 \%$ e $1 \%$.

Pelos valores obtidos, percebe-se que o coeficiente que afere a persistência do desempenho superior $\left(L E F_{t-1}\right)$ apresentou sinal positivo e significativo a pelo menos $5 \%$ para todos os setores analisados. Tal evidência converge para os achados de Carvalho et al. (2010) e indica que parte do resultado do lucro específico da firma é transmitido para o período posterior.

A tangibilidade, medida pelo Varbook, manifestou-se significativa a 5\% e positiva para os setores de Bens Industriais, Construção e Transporte, Consumo Não Cíclico, Materiais Básicos e Utilidade Pública. Nos demais, não foi possível observar qualquer efeito da tangibilidade sobre o desempenho. Isto converge em parte para os achados de Carvalho et al. (2010), em que as evidências apontam para a significância da medida de tangibilidade em todos os setores analisados.

Esses resultados corroboram os fundamentos da RBV. Para Peteraf (1993), a posse ou disposição de recursos não asseguram vantagem competitiva sustentável às firmas. A performance mais elevada está associada à superioridade dos recursos face aos seus competidores e concorrentes.

Os setores tangíveis-intensivos (com médias setoriais de Varbook acima da média geral) apresentaram coeficiente negativo e significativo para a medida de tangibilidade. Isto implica que, para os setores de Bens Industriais, Construção e Transporte, Consumo Não Cíclico, Materiais Básicos e Utilidade Pública, a tangibilidade concorre para reduzir a persistência do desempenho superior das companhias. Os setores de Consumo Cíclico e Telecomunicações e Tecnologia (setores intangíveisintensivos), com menor média de Varbook, exibiram coeficientes positivos e significativos no desempenho superior das companhias. 
Para o mercado brasileiro, estudos de Brito e Vasconcelos (2004) e Carvalho et al. (2010) indicavam que aumentos na tangibilidade de ativos proporcionam menores níveis de persistência no desempenho específico das firmas. Ainda, os achados se alinham às evidências em Schmalensee (1985), Titman e Wessels (1988), Rumelt (1991), Mauri e Michaels (1998), Chang e Singh (2000), Chang e Hong (2003) e Hawawini, Subramanian e Verdin (2003).

Uma explicação para as diferenças dos resultados da sustentabilidade do desempenho superior reside na ideia de que não apenas os recursos são estratégicos para as firmas tratadas isoladamente, mas também o são em setores da economia. Essa tese é reforçada pelas posições de Hunt e Morgan (1995), Adner e Zenski (2006), Chareonsuk e Chansa-Ngavej (2008) e Madhani (2009), por defenderem o efeito heterogêneo dos recursos na sustentabilidade do desempenho econômico.

Os dados exibem um efeito significativo a $1 \%$ e positivo do coeficiente da responsabilidade social sobre o lucro específico superior nos setores tangível-intensivos. Percebem-se os maiores coeficientes alocados nos setores que fazem uso mais intensivo da tangibilidade. No Brasil, as evidências se alinham aos achados de M. A. V. Machado e Machado (2011), ao encontrar efeitos positivos da responsabilidade social, internos e externos, sobre a rentabilidade das companhias. Entretanto, do ponto de vista do efeito da responsabilidade social sobre a persistência do desempenho superior, esta é significativa para todos os setores. Neste ponto, sugere-se que a responsabilidade social tenha um efeito mais homogêneo para a sustentação da vantagem competitiva do que na geração do desempenho corrente.

A variável de Governança (Govnce) apresentou significância estatística a $1 \%$ para os setores intensivo-tangíveis (Bens Industriais, Construção e Transporte e Consumo Não Cíclico). Isto implica que, para esses setores, o nível de governança promove resultado superior. Para as companhias dos outros setores (a maioria intangíveis-intensivos), os níveis de governança parecem não apresentar vantagens competitivas por razão da governança. Entretanto, os mesmos setores (Bens Industriais, Construção e Transporte e Consumo Não Cíclico), que se beneficiam da governança como mecanismo de geração de desempenho corrente, apresentam significância estatística a 1\% com sinal negativo para a variável de interação Govnce $x \mathrm{LEF}_{\mathrm{t}-1 .}$. Isto significa que há evidências de que os níveis de governança impactam, negativamente, a persistência do desempenho superior das companhias. Neste sentido, se por um lado a governança promove resultados positivos para geração de valor em um dado tempo, concorre para redução da persistência desse valor no longo prazo. Tal característica chama a atenção para a consistência e dinâmica de suas práticas ao longo do tempo. Os achados sugerem efeitos temporários da governança sobre os resultados das companhias.

\section{Qualidade e especificação dos modelos}

Os testes de Sargan e de correlação serial foram empregados para verificar a validade da especificação dinâmica do modelo, onde, além do efeito da persistência do desempenho, introduziramse defasagens nos regressores, tornando necessário o uso de instrumentos para identificar as variáveis endógenas.

Por meio desse procedimento, é possível notar a hipótese nula de que a correlação entre os erros e os instrumentos é zero. Dessa forma, a falha em rejeitar a hipótese nula (probabilidades do teste Sargan acima de 5\%) indicou que os instrumentos são robustos. Já o teste de correlação serial investiga a hipótese de que os erros são serialmente não correlacionados. Os resultados evidenciam que os erros em primeira diferença $[\mathrm{AR}(1)]$ são correlacionados em primeira ordem e não correlacionados em segunda[AR(2)], indicando que os instrumentos utilizados não se relacionam com o termo de erro.

A ordem das defasagens utilizadas para a variável LEF em ambas as equações (equações 3 e 4) baseou-se no menor valor de AIC (Critério de Informação de Akaike), conforme orienta Wooldridge (2010), bem como no teste de autocorrelação de segunda ordem AR(2) (Greene, 2003). Neste sentido, para todos os setores, os modelos GMM-SYS foram implementados para um lag de diferença para LEF, em consequência de não haver problemas com autocorrelação de segunda ordem nos resíduos desses modelos, além de apresentar menor valor de AIC entre os modelos com 2 e 3 lags para $\triangle \mathrm{LEF}$. 
Os modelos foram implementados por GMM-SYS de dois estágios que, segundo Windmeijer (2005), são mais eficientes. Para isso, o autor sugere a correção da matriz de variância/covariância de maneira a conduzir estimativas de erros robustos. Neste sentido, efetuou-se GMM-SYS com erros robustos, após a aferição das estimativas GMM, para efeito do teste Sargan. Ressalta-se que, sob a hipótese de não normalidade nos erros do GMM-SYS, o uso de um vetor de correção de erros torna dispensável o teste sobreidentificação de Sargan (Windmeijer, 2005).

Assim, os modelos GMM-SYS apresentaram-se consistentes e bem especificados, uma vez que o teste de adequação do modelo foi significativo para um nível de probabilidade de $1 \%$ (Prob $\left.>\mathrm{Chi}^{2}=0,0000\right)$, rejeitando a hipótese nula de que o efeito conjunto das variáveis fosse nulo.

Wooldridge (2010) aponta para a necessidade de inspecionar a estacionaridade nas análises com séries em painel. Neste ponto, a estacionaridade torna-se condição necessária para a implementação dos modelos, com variáveis em primeiras diferenças. Quando não estacionária, não deve compor o set de variáveis explicativas do modelo de regressão. Neste sentido, aplicaram-se os seguintes testes para raiz unitária em dados em painel: Levin, Li Shu (LCC), que assumem processo comum de raiz unitária; e os testes de Im, Pesaram e Shim (IPS), que declaram processo individual de raiz unitária. Os testes foram realizados para as séries em nível utilizando-se do critério de seleção para o número de defasagens de Hannan-Quinn. Todas as variáveis (LEF, QTobin, Varbook, ESG, Govnce, e as de interação), em todos os setores, rejeitaram a hipótese nula para os testes LCC e IPS (que constatam Ho para a presença de raiz unitária comum, no caso do teste LCC, e raiz unitária individual no de IPS). Por conseguinte, todas as variáveis são estacionárias para todos os setores analisados.

A inspeção acerca da heterocedasticidade seguiu a proposição apresentada por Grenne (2003), a partir do teste modificado de Wald, que formaliza como hipótese nula a homocedasticidade nos termos de erro. Os resultados apontaram para a rejeição da hipótese nula, a um nível de significância de 1\%, para todos os setores examinados, demonstrando que os erros dos modelos são heterocedásticos. Assim sendo, estes foram reespecificados utilizando-se estimativas robustas para os termos de erro dos modelos GMM-SYS, já recomendadas por Windmeijer (2005).

\section{Considerações Finais}

Pesquisar os fatores relacionados à causalidade do desempenho superior das empresas tem promovido relevantes discussões empírico-teóricas. A RBV segue um mainstream de que as corporações modelam uma combinação apropriada dos seus recursos, de forma heterogênea, em cada setor de atuação, devido ao dinamismo dos processos e aos limites que são impostos na capacidade produtiva. Destarte, os achados na pesquisa trazem algumas questões importantes.

O primeiro destaque refere-se às evidências da contribuição da intangibilidade como mecanismo de redução da persistência do desempenho superior para a grande maioria dos setores, no Brasil, com exceção para o setor de Utilidade Pública. Para este último, maiores níveis de intangibilidade tendem a contribuir com a persistência do desempenho superior de forma significativa a $1 \%$ e de acordo com as evidências em Villalonga (2004), sendo estes resultados parcialmente distintos de Carvalho et al. (2010), que encontraram uma relação negativa e significativa para todos os setores analisados, com exceção para os setores de agro/pesca e transporte/serviços (embora não significativos do ponto de vista da inferência estatística, apresentaram sinais negativos para seus coeficientes nas subamostras analisadas).

O segundo dado trazido pela pesquisa foi de que a tangibilidade demonstrou comportamento heterogêneo sobre a persistência do desempenho superior nas companhias abertas brasileiras. Sob esta premissa, as evidências corroboraram os achados em Carvalho et al. (2010). Viu-se que os setores de Bens Industriais, Construção e Transporte, Materiais Básicos e Consumo Não Cíclico apresentaram efeito negativo da tangibilidade sobre a persistência do desempenho superior das companhias. 
Destacam-se as evidências para o setor de Tecnologia e Telecomunicações que apresentou sinal positivo, divergindo de Carvalho et al. (2010).

O terceiro elemento fundamental notado faz alusão às evidências do efeito significativo e positivo do nível de responsabilidade social sobre o desempenho e persistência do desempenho superior das empresas de capital aberto no Brasil. Neste aspecto, a responsabilidade social parece promover um papel de destaque na formação da vantagem competitiva das companhias. Entretanto, a governança parece ter um efeito particular e heterogêneo, tanto para a formação do desempenho corrente, quanto para a persistência do desempenho superior, seja como variável de controle para o modelo da intangibilidade, seja para o modelo de tangibilidade.

É importante ressaltar que, nesta investigação, admitiu-se uma definição uniforme para a tangibilidade dos ativos, pressupondo que todos os bens de uma empresa possuam igual capacidade de construir a competitividade e a persistência dos resultados. Evidentemente, esse pressuposto é bastante restritivo e pode não representar de forma mais eficiente as relações da tangibilidade com a persistência do desempenho financeiro das empresas. De maneira equivalente, os indicadores de governança e responsabilidade social utilizados representam generalizações acerca das características mais específicas das companhias. Assim, é importante limitar os achados dessa investigação sob as condições aqui impostas. Entretanto, as evidências sugerem, mesmo que de forma genérica, observar uma relação de dependência, sobretudo da variável responsabilidade social sobre a persistência do desempenho das companhias abertas no Brasil.

Notoriamente, sugere-se que futuras investigações possam tratar a tangibilidade de forma clusterizada por perfil de ativo. De forma semelhante, sugere-se o desmembramento das métricas de governança e responsabilidade social nas suas dimensões idiossincráticas. É possível que essas novas variáveis possam elucidar, de forma ainda mais transparente, e conferir melhor resposta sobre os indutores da persistência do desempenho em discussão.

\section{Referências}

Adner, R., \& Zemsky, P. (2006). A demand-based perspective on sustainable competitive advantage. Strategic Management Journal, 27(3), 215-239. http://dx.doi.org/10.1002/smj.513

Arellano, M., \& Bond, S. (1991). Some tests of specification for panel data: Monte Carlo evidence and an application to employment equations. Review of Economic Studies, 58(2), 277-297. http://dx.doi.org/10.2307/2297968

Arellano, M., \& Bover, O. (1995). Another look at the instrumental variable estimation of errorcomponents models. Journal of Econometrics, 68(1), 29-51. http://dx.doi.org/10.1016/03044076(94)01642

Baltagi, B. H. (2008). Econometric analysis of panel data (4th ed.). Weston Sussex: John Miley \& Sons.

Barney, J. (1991). Firm resources and sustained competitive advantage. Journal of Management, 17(1), 99-120. http://dx.doi.org/10.1177/014920639101700108

Barney, J., \& Hesterly, W. S. (2007). Administração estratégica e vantagem competitiva. São Paulo: Pearson Prentice Hall.

Bianchi, P., \& Labory, S. (2004). The economic importance of intangible assets. Burlington: Ashgate.

Blundell, R., \& Bond, S. (1998). Initial conditional and moment restritions in dynamic panel data models. Journal of Econometrics, 87(1), 115-143. http://dx.doi.org/10.1016/S03044076(98)00009-8 
Brammer, S., Brooks, C., \& Pavelin, S. (2006). Corporate social performance and stock returns: UK evidence from disaggregate measures. Financial Management, 35(3), 97-116. http://dx.doi.org/10.1111/j.1755-053X.2006.tb00149.x

Brito, L. A. L., \& Vasconcelos, F. C. de (2004). A heterogeneidade do desempenho, suas causas e o conceito de vantagem competitiva: proposta de uma métrica [Edição Especial]. Revista de Administração Contemporânea, $\quad 8, \quad 107-129 . \quad$ Recuperado de http://www.scielo.br/pdf/rac/v8nspe/v8nespa07.pdf. $\quad$ http://dx.doi.org/10.1590/S141565552004000500007

Burton, B. M., Lonie, A. A., \& Power, D. M. (1999). The stock market reaction to investiment announcements: the case of individual capital expenditure projects. Journal of Business Finance and Accounting, 26(6), 681-708. http://dx.doi.org/10.1111/1468-5957.00271

Carmeli, A., \& Tishler, A. (2004). The relationships between intangible organizational elements and organizational performance. Strategic Management Journal, 25(13), 1257-1278. http://dx.doi.org/10.1002/smj.428

Carvalho, F. de M., Kayo, E. K., \& Martin, D. M. L. (2010). Tangibilidade e intangibilidade na determinação do desempenho. Revista de Administração Contemporânea, 14(5), 871-889. Recuperado de http://www.scielo.br/pdf/rac/v14n5/v14n5a07.pdf. http://dx.doi.org/10.1590/S1415-65552010000500007

Catapan, A., \& Colauto, R. D. (2014). Governança corporativa: uma análise de sua relação com o desempenho econômico-financeiro das empresas cotadas no Brasil nos anos de 2010-2012. Contadoria y Administracion, 59(3), 137-164. http://dx.doi.org/10.1016/S0186-1042(14)71268-9

Chang, S. J., \& Hong, J. (2003). How much does the business group matter in Korea? Strategic Management Journal, 23(3), 263-274. http://dx.doi.org/10.1002/smj.224

Chang, S. J., \& Singh, H. (2000). Corporate and industry effects on business unit competitive position. Strategic Management Journal, 21(7), 739-752. http://dx.doi.org/10.1002/10970266(200007)21:7<739::AID-SMJ117>3.0.CO;2-Q

Chareonsuk, C., \& Chansa-Ngavej, C. (2008). Intangible asset management framework for long-term financial performance. Industrial Management and Data Systems, 108(6), 812-828. http://dx.doi.org/10.1108/02635570810884021

Chung, K. H., Wright, P., \& Charoenwong, C. (1999). Investiment oportunities and market reaction to capital expenditure decisions. Journal of Banking and Finance, 22(1), 41-60. http://dx.doi.org/10.1016/S0378-4266(97)00021-6

Cohen, J. A. (2005). Intangible assets: valuation and economic benefit. New York: J. Wiley.

Colauto, R. D., Nascimento, P. S., Avelino, B. C., \& Bispo, O. N. A. (2009). Evidenciação de ativos intangíveis não adquiridos nos relatórios da administração das companhias listadas nos níveis de governança corporativa da Bovespa. Contabilidade Vista e Revista, 20(1), 142-169.

Daniel, K., \& Titman, S. (2006). Market reactions to tangible and intangible information. Journal of Finance, 61(4), 1605-1643. http://dx.doi.org/10.1111/j.1540-6261.2006.00884.x

Danthine, J. P., \& Jin, X. (2007). Intangible capital, corporate valuation and asset pricing. Economic Theory, 32(1), 157-177. http://dx.doi.org/10.2139/ssrn.932046

Daun, J. H. (2003). Intangible assets and value creation. New York: Wiley \& Sons.

Fama, E., \& French, K. R. (1993). Common risk factors in the returns on stocks and bonds. Journal of Financial Economics, 33(1), 3-56. http://dx.doi.org/10.1016/0304-405X(93)90023-5 
Ferreira, D. A., Ávila, M., \& Faria, M. D. (2010). Efeitos da responsabilidade social corporativa na intenção de compra e no benefício percebido pelo consumidor: um estudo experimental. Revista de Administração, 45(3), 285-296. http://dx.doi.org/10.1590/S0080-21072010000300007

Freguette, L. M., Nossa, V., \& Funchal, B. (2014). Responsabilidade social corporativa e desempenho financeiro das empresas brasileiras na crise de 2008. Revista de Administração Contemporânea, 19(2), 232-248. Recuperado de http://www.scielo.br/pdf/rac/v19n2/1415-6555-rac-19-0200232.pdf. http://dx.doi.org/10.1590/1982-7849rac20151873

Glick, W. H., Washburn, N. T., \& Miller, C. C. (2005, May). The myth of firm performance. Proceedings of the Annual Meeting of the Academy of Management, Honolulu, HI, USA, 70. http://dx.doi.org/10.1287/orsc.1120.0762

Greene, W. H. (2003). Econometric analysis (7th ed.). New York: Hardcover.

Hawawini, G., Subramanian, V., \& Verdin, P. (2003). Is performance driven by industry - or firm specific factors? A new look at the evidence. Strategic Management Journal, 24(1), 1-16. http://dx.doi.org/10.1002/smj.278

Hunt, S. D., \& Morgan, R. M. (1995). The comparative advantage theory of competition. Journal of Marketing, 59(4), 1-15. http://dx.doi.org/10.2307/1252087

Kapler, L., \& Love, L. (2002). Corporate governance, investor protection and performance in emerging markets [Working Paper $\mathrm{n}^{\circ}$ 2818]. World Bank Policy Research, Washington, USA. http://dx.doi.org/10.2139/ssrn.303979

Kayo, E. K., Kimura, H., Martin, D. M. L., \& Nakamura, W. T. (2006). Ativos intangíveis, ciclo de vida e criação de valor. Revista de Administração Contemporânea, 10(3), 73-90. Recuperado de http://www.scielo.br/pdf/rac/v10n3/a05v10n3.pdf. 65552006000300005

Kunc, M. H., \& Morecroft, J. D. W. (2010). Managerial decision-making and firm performance under a resource-based paradigm. Strategic Management Journal, 31(11), 1164-1180. http://dx.doi.org/10.1002/smj.858

Laan, G. V. D., Ees, H. V., \& Witteloostuijn, A. V. (2008). Corporate social and finance performance: an extended stakeholder theory, and empirical test with accounting measures. Journal of Business Ethics, 79(3), 299-310. http://dx.doi.org/10.1007/s10551-007-9398-0

Leal, R. P. (2004). Governance practices and corporate value: a recent literature survey. Revista de Administração de Empresas da USP, 39(4), 327-337. http://dx.doi.org/10.1590/S167869712013000400006

Leal, R. P. C., \& Carvalhal da Silva, A, L. (2007). Corporate governance and value in Brazil (and in Chile). In A. Chong \& F. Lopez-de-Silanes (Orgs.), Investor protection and corporate governance: firm level evidence across Latin American (pp. 213-287). Palo Alto: Stanford University Press.

Lev, B. (2001). Intangibles: management, measurement and reporting. Washington: Brookings Institution.

Lev, B., \& Zambon, S. (2003). Intangible and intelectual capital: an introduction to a special issue. European Accounting Review, 12(4), 597-603. http://dx.doi.org/10.1080/0963818032000162849

Lin, G. T. R., \& Tang, J. Y. H. (2009). Appraising intangible assets from the viewpoint of value drivers. Journal of Business Ethics, 88(4), 679-689. http://dx.doi.org/10.1007/s10551-008-9974-y 
Lockett, A., Morgenstern, U., \& Thompson, S. (2009). The development of the resource-based view of the firm: a critical appraisal. International Journal of Management, 11(1), 9-28. http://dx.doi.org/10.1111/j.1468-2370.2008.00252.x

Lucchesi, E. P., \& Famá, R. (2007). O impacto das decisões de investimento das empresas no valor de mercado das ações negociadas na Bovespa no período de 1996 a 2000. Revista de Administração, 42(2), 249-260. http://dx.doi.org/10.1590/S1678-69712012000400004

Lyra, R., \& Olinquevitch, J. (2007). Análise do conteúdo informacional dos investimentos em ativo imobilizados: um estudo de eventos em empresas negociadas na Bovespa. Revista Universo Contábil, 3(2), 39-53.

Machado, M. A. V., \& Machado, M. R. (2011). Responsabilidade social impacta o desempenho financeiro das empresas? Advances and Applied Accounting, 4(1), 2-23. http://dx.doi.org/10.14392/ASAA.2011040101

Machado, M. R., Machado, M. A. V., \& Corrar, L. J. (2009). Desempenho do índice de sustentabilidade empresarial (ISE) da bolsa de valores de São Paulo. Revista Universo Contábil, 5(2), 24-38. http://dx.doi.org/10.4270/ruc.2009211

Mackey, A., Mackey, T. B., \& Barney J. B. (2005). Corporate social responsability and firm performance: investor preferences and corporate strategies. Academy of Management Review, 2(26), 2-14. http://dx.doi.org/10.5465/AMR.2007.25275676

Madhani, P. M. (2009). Bankruptcy of lehman brothers: a pointer of subprime crisis. The Accounting World, 9(6), 33-39.

Mathews, J. A. (2002). A resource-based view of Schumpeterian economic dynamics. Journal of Evolutionary Economics, 12(1), 29-54. http://dx.doi.org/10.1007/2F978-3-7908-2720-0

Mauri, A. J., \& Michaels, M. P. (1998). Firm and industry effects within strategic management: an empirical examination. Strategic Management Journal, 19(3), 211-219. http://dx.doi.org/10.1002/(SICI)1097-0266(199803)19:3

Mcconnel, J. J., \& Muscarella, C. J. (1985). Corporate capital expenditure decisions and the market value of the firm. Journal of Financial Economics, 14(3), 399-482. http://dx.doi.org/10.1016/0304-405X(85)90006-6

Orlitzky, M. (2001). Corporate social performance and firm risk: a meta-analytic review. Business \& Society, 40(4), 369-396. http://dx.doi.org/10.1177/000765030104000402

Pava, M. L., \& Krausz, J. (1996). The association between corporate social-responsability and financial performance: the paradox of social cost. Journal of Business Ethics, 15(3), 321-357. http://dx.doi.org/10.1007/2FBF00382958

Pavão, Y., Sehnem, S., \& Hoffmann, V. E. (2011). Análise dos recursos organizacionais que sustentam a vantagem competitiva. Revista de Administração, 46(3), 228-242. http://dx.doi.org/10.5700/rausp1009

Peteraf, M. A. (1993). The cornerstones of competitive advantage: a resource-based view. Strategic Management Journal, 14(3), 179-191. http://dx.doi.org/10.1002/smj.4250140303

Rumelt, R. P. (1991). How much does industry matter? Strategic Management Journal, 12(3), 167-185. http://dx.doi.org/10.1002/(SICI)1097-0266(199707)18

Santos, D. F. L., Basso, L. F. C., Kimura, H., \& Kayo, E. K. (2014). Innovation efforts and performances of Brazilian firms. Journal of Business Research, 67(1), 527-535. http://dx.doi.org/10.1016/j.jbusres.2013.11.009 
Schmalensee, R. (1985). Do markets differ much? American Accounting Review, 75(3), 341-351.

Shleifer, A., \& Vishny, R. (1997). A survey of corporate governance. Journal of Finance, 52(2), 737783. http://dx.doi.org/10.3386/w5554

Surroca, J., Tribó, J. A., \& Waddock, S. (2010). Corporate responsability and financial performance: the role of intangible resources. Strategic Management Journal, 31(5), 463-490. http://dx.doi.org/10.1002/smj.820

Titman, S., \& Wessels, R. (1988). The determinants of capital structure choice. The Journal of Finance, 43(1), 1-19. http://dx.doi.org/10.1111/j.1540-6261.1988.tb02585.x

Villalonga, B. (2004). Intangible resources, Tobin's Q, and sustainability of performance differences. Journal of Economic Behavior \& Organization, 54(2), 205-230. http://dx.doi.org/10.1016/j.jebo.2003.07.001

Wernerfelt, B. A. (1984). A resource-based view of the firm. Strategic Management Journal, 5(2), 171179. http://dx.doi.org/10.1002/smj.4250160303

Windmeijer, F. (2005). A finite sample correction for the variance of linear efficient two-step GMM estimators. Journal of Econometrics, 126(1), 25-51. http://dx.doi.org/10.1920/wp.ifs.2000.0019

Wood, D. J. (1991). Corporate social performance revisited. The Academy of Management Review, 16(4), 691-718. http://dx.doi.org/10.5465/AMR.1991.4279616

Wooldridge, J. M. (2010). Solutions manual and supplementary materials for econometric analysis of cross section and panel data (2nd ed.). Cambridge: MIT Press Books.

\section{Dados dos Autores}

Ayron Wanderley Medeiros

Av. Amintas Barros, 3003, 59075-250, Natal, RN, Brasil. E-mail: ayronmedeiros@ @otmail.com

Anderson Luiz Rezende Mol

Edifício do Centro de Ciências Sociais Aplicadas Campus Universitário, Lagoa Nova, Caixa Postal 1620, 59072-970, Natal, RN, Brasil. E-mail: mol.ufrn@gmail.com 